International Journal of Pure and Applied Mathematics

Volume 95 No. 4 2014, 493-502

ISSN: 1311-8080 (printed version); ISSN: 1314-3395 (on-line version)

url: http://www.ijpam.eu

doi: http://dx.doi.org/10.12732/ijpam.v95i4.1

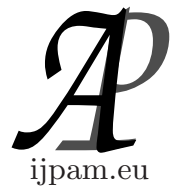

\title{
HOPF BIFURCATION AND GLOBAL PERIODIC SOLUTION \\ IN A DELAYED STAGE-STRUCTURED \\ PREY-PREDATOR SYSTEM
}

\author{
Shunyi $\mathrm{Li}^{1}{ }^{\S}, \mathrm{Yu} \mathrm{Wei}^{2}$, Wenwu $\mathrm{Liu}^{3}$ \\ 1,2,3 Department of Mathematics \\ Qiannan Normal College for Nationalities \\ Duyun, Guizhou, 558000, P.R. CHINA
}

\begin{abstract}
A prey-predator model with stage-structured for predator and time delay is considered. The characteristic equations of the equilibrium points are analyzed, by applying the theorem of Hopf bifurcation, the conditions for the positive equilibrium occurring local Hopf bifurcation are given. The conditions for the existence of global Hopf bifurcation of the system are obtained. Finally, numerical simulation and brief conclusion are given.
\end{abstract}

AMS Subject Classification: 34K13, 34K18, 34K20, 34K60, 92D25

Key Words: prey-predator system, stage-structured, time delay, Hopf bifurcation, global periodic solution

\section{Introduction}

In population dynamics, time delays play an important role, which can make the system's equilibrium points loss of its stability and bifurcate various periodic solutions, even chaos. Recently, S.Y. Li and X.G. Xue [1, 2] investigated the local Hopf bifurcation of a three-stage-structured prey-predator system, they took time delay $\tau$ as the bifurcation parameter, shown that, the positive equilibrium loses its stability and a local Hpof bifurcation occurring when the

Received: $\quad$ March 31, 2013

(C) 2014 Academic Publications, Ltd.

${ }^{\S}$ Correspondence author url: www.acadpubl.eu 
delay $\tau$ passes through the first critical value $\tau_{0}$.

In delayed population systems, are there existence of large-scale periodic solutions (global Hopf bifurcation) when $\tau$ far away from the first Hopf bifurcation critical values $\tau_{0}$ ? C.J. Sun, M.A. Han and Y.P. Lin [3] studied the global Hopf bifurcation of a delayed logistic model by using a the result due to Wu [4]. The global periodic solutions of a delayed predator-prey system have considered by X.P. Yan and W.T. Li [5]. In this paper, we consider the Hopf bifurcation and global periodic solution of following stage-structured prey-predator system with hunting delay

$$
\left\{\begin{array}{l}
x_{1}^{\prime}(t)=\alpha x_{2}(t)-x_{1}(t)\left[\gamma-\eta x_{1}(t)-E y(t-\tau)\right] \\
x_{2}^{\prime}(t)=\Omega x_{1}(t)-\theta x_{2}(t) \\
x_{3}^{\prime}(t)=a x_{2}(t)-b x_{3}(t)-c x_{3}^{2}(t) \\
y^{\prime}(t)=y(t)\left(k E x_{1}(t)-d-f y(t)\right)
\end{array}\right.
$$

where $\gamma=\gamma_{1}+\Omega, \theta=\theta_{1}+a . x_{i}(t)(i=1,2,3)$ are the densities of immature preys, mature preys and old preys at time $t, y(t)$ is the density of predator at time $t$, respectively. All of the parameters are positive, $\alpha$ is the birth rate of mature prey population, and $\gamma_{1}, \theta_{1}, b$ are the death rate of immature, mature and old prey population, respectively. $\Omega$ and $a$ are the maturity rate and ageing rate of the prey population, respectively. $\eta, c$ and $f$ are the density dependent coefficients of immature and old prey population, predator population, respectively. $k(0<k<1)$ is the rate of conversing prey into predator and $E$ is the predation coefficient. $\tau$ is the hunting delay for predator population. The initial conditions for (1) are:

$$
x_{i}(t)=\phi_{i}(i) \geq 0(i=1,2,3), y(t)=\varphi(t) \geq 0, t \in[-\tau, 0] .
$$

\section{Local Hopf Bifurcation}

Obviously, (1) has two boundary equilibrium $E_{0}=(0,0,0,0)$ and $E_{1}\left(x_{1}, x_{2}, x_{3}, 0\right)$ (if condition $C_{1}: \alpha \Omega-\gamma \theta>0$ holds), and a unique positive equilibrium $E_{2}\left(x_{1}^{*}, x_{2}^{*}, x_{3}^{*}, y^{*}\right)$ (if condition $C_{2}: k E x_{1}^{*}-d>0$ holds), where

$$
\begin{gathered}
x_{1}=\frac{\alpha \Omega-\gamma \theta}{\eta \theta}, x_{2}=\frac{\Omega}{\theta} x_{1}, x_{3}=\frac{\sqrt{b^{2}+4 a c x_{2}}-b}{2 c}=f\left(x_{2}\right), \\
x_{1}^{*}=\frac{f(\alpha \Omega-\gamma \theta)+d E \theta}{\theta\left(k E^{2}+\eta f\right)}, x_{2}^{*}=\frac{\Omega}{\theta} x_{1}^{*}, x_{3}^{*}=f\left(x_{2}^{*}\right), y^{*}=\frac{k E x_{1}^{*}-d}{f} .
\end{gathered}
$$


The characteristic equation about the positive equilibrium $E_{2}$ is given by

$$
\operatorname{det}\left(\begin{array}{cccc}
a_{11} & \alpha & 0 & -E x_{1}^{*} e^{-\lambda \tau} \\
\Omega & -\theta-\lambda & 0 & 0 \\
0 & a & -b-2 c x_{3}^{*}-\lambda & 0 \\
k E y^{*} & 0 & 0 & -f y^{*}-\lambda
\end{array}\right)=0
$$

where $a_{11}=-\gamma-2 \eta x_{1}^{*}-E y^{*}-\lambda$. Namely, $\left(\lambda+b+2 c x_{3}^{*}\right) D(\lambda, \tau)=0 . \quad \lambda_{4}=$ $-b-2 c x_{3}^{*}<0, \lambda_{i}(i=1,2,3)$ are the roots of the characteristic equation

$$
D(\lambda, \tau)=M(\lambda)+N(\lambda) e^{-\lambda \tau}=0,
$$

where

$$
\begin{aligned}
& M(\lambda)=\lambda^{3}+m_{2} \lambda^{2}+m_{1} \lambda+m_{0}, N(\lambda)=n_{1} \lambda+n_{0} \\
& m_{2}=\gamma+2 \eta x_{1}^{*}+E y^{*}+\theta+f y^{*}, \\
& m_{1}=\theta\left(\eta x_{1}^{*}+f y^{*}\right)+f y^{*}\left(\gamma+2 \eta x_{1}^{*}+E y^{*}\right), \\
& m_{0}=f \eta \theta x_{1}^{*} y^{*}, n_{1}=k E^{2} x_{1}^{*} y^{*}, n_{0}=k E^{2} \theta x_{1}^{*} y^{*} .
\end{aligned}
$$

When $\tau=0$, (2) becomes

$$
\lambda^{3}+m_{2} \lambda^{2}+\left(m_{1}+n_{1}\right) \lambda+m_{0}+n_{0}=0 .
$$

Note that, if $C_{3}: k E^{2}<f \eta$ hold true, then $m_{2}\left(m_{1}+n_{1}\right)-\left(m_{0}+n_{0}\right)>0$. By Routh-Hurwits criterion, all roots of (3) have negative real parts. Then, the equilibrium $E_{2}$ is local stable.

Suppose $\lambda=i \omega(\omega>0)$ is a root of (2) and separating the real and imaginary parts, we have

$$
\left\{\begin{array}{l}
m_{2} \omega^{2}-m_{0}=\left(n_{0}-n_{2} \omega^{2}\right) \cos \omega \tau+n_{1} \omega \sin \omega \tau \\
\omega^{3}-m_{1} \omega=n_{1} \omega \cos \omega \tau-\left(n_{0}-n_{2} \omega^{2}\right) \sin \omega \tau
\end{array}\right.
$$

From (4), one can get that

$$
\left(n_{0}-n_{2} \omega^{2}\right)^{2}+n_{1}^{2} \omega^{2}=\left(m_{2} \omega^{2}-m_{0}\right)^{2}+\left(\omega^{3}-m_{1} \omega\right)^{2} .
$$

Namely

$$
F(\omega)=\omega^{6}+p \omega^{4}+q \omega^{2}+r=0,
$$

where $p=m_{2}^{2}-2 m_{1}-n_{2}^{2}>0, q=m_{1}^{2}+2 n_{2} n_{0}-n_{1}^{2}-2 m_{2} m_{0}$,

$$
r=m_{0}^{2}-n_{0}^{2}=x_{1}^{*} y^{*} \theta\left(m_{0}+n_{0}\right)\left(k E^{2}-f \eta\right) .
$$

If $C_{3}: k E^{2}<f \eta$ and $q>0$ hold, we know that (6) has a unique positive root $\omega_{0}$. From (4), we have

$$
\cos \omega_{0} \tau=\frac{\left(m_{2} \omega_{0}^{2}-m_{0}\right)\left(n_{0}-n_{2} \omega_{0}^{2}\right)+n_{1} \omega_{0}\left(\omega_{0}^{3}-m_{1} \omega_{0}\right)}{\left(n_{0}-n_{2} \omega_{0}^{2}\right)^{2}+\left(n_{1} \omega_{0}\right)^{2}},
$$


Thus

$$
\begin{aligned}
\tau_{j}= & \frac{1}{\omega_{0}} \cos ^{-1}\left[\frac{\left(m_{2} \omega_{0}^{2}-m_{0}\right)\left(n_{0}-n_{2} \omega_{0}^{2}\right)+n_{1} \omega_{0}\left(\omega_{0}^{3}-m_{1} \omega_{0}\right)}{\left(n_{0}-n_{2} \omega_{0}^{2}\right)^{2}+\left(n_{1} \omega_{0}\right)^{2}}\right] \\
& +\frac{2 j \pi}{\omega_{0}}, j=0,1,2, \cdots
\end{aligned}
$$

Let $\lambda(\tau)=v(\tau)+\mathrm{i} \omega(\tau)$ be the roots of (2) such that when $\tau=\tau_{j}$ satisfying $v\left(\tau_{j}\right)=0$ and $\omega\left(\tau_{n}\right)=\omega_{0}$. Since $\omega_{0}$ is the unique positive root of $(6)$, then, at each $\tau=\tau_{j}(j=0,1,2, \cdots)$ straightforward calculation gives [6]

$$
\operatorname{sign}\left[\mathrm{d} F(\omega) /\left.\mathrm{d} \omega\right|_{\omega=\omega_{0}}\right]=2 \omega_{0}\left(3 \omega_{0}^{4}+2 p \omega_{0}^{2}+q\right)>0 .
$$

According to the Hopf bifurcation theorem for functional differential equations [7], we have the following result.

Theorem 1. If $C_{3}: k E^{2}<f \eta$ and $q>0$ hold, then there exists $\tau_{0}$, when $\tau \in\left[0, \tau_{0}\right)$ the positive $E_{2}$ of $(1)$ is asymptotically stable and unstable when $\tau>\tau_{0}$; system (1) can undergo a local Hopf bifurcation near the positive equilibrium $E_{2}$ when $\tau=\tau_{j}(j=0,1,2, \cdots)$.

\section{Global Hopf Bifurcation}

In this section, we study the existence of global Hopf bifurcations. The method we used here is based on the global Hopf bifurcating theorem for general functional differential equations introduced by $\mathrm{Wu}$ [4]. For convenience, we write system (1) as the following form

$$
z^{\prime}(t)=F\left(z_{t}, \tau\right)
$$

where $z(t)=\left(x_{1}(t), x_{2}(t), x_{3}(t), y(t)\right), z_{t}(\theta)=z(t+\theta) \in\left([-\tau, 0], \mathbb{R}^{4}\right)$.

Define $X=C\left([-\tau, 0], \mathbb{R}^{4}\right), \Sigma=C l\left\{(z(t), \tau, T) \in X \times \mathbb{R} \times \mathbb{R}^{+}, z(t)\right.$ is $T$ periodic solution of $(1)\}, N=\{(z(t), \tau, T), F(z(t), \tau, T)=0\}$.

Let $l_{\left(E_{2}, \tau_{j}, 2 \pi / \omega_{0}\right)}$ be the connected component $\left(E_{2}, \tau_{j}, 2 \pi / \omega_{0}\right)$ in $\Sigma$, where $\tau_{j}$ and $\omega_{0}$ defined in (8).

Lemas 2. All solution of (1) are positive and uniformly bounded.

Proof. It is true that,

$$
\begin{aligned}
& \left.x_{1}^{\prime}(t)\right|_{x_{1}(t)=0}=\alpha x_{2}(t) \geq 0, \\
& \left.x_{2}^{\prime}(t)\right|_{x_{2}(t)=0}=\Omega x_{1}(t) \geq 0,\left.x_{3}^{\prime}(t)\right|_{x_{3}(t)=0}=a x_{2}(t) \geq 0, \\
& y(t)=\varphi(0) \exp \left\{\int_{0}^{t}\left[k E x_{1}(s)-d-f y(s)\right] \mathrm{d} s\right\} \geq 0 .
\end{aligned}
$$


Let $V(t)=\sum_{i=1}^{3} x_{i}(t)$, for $0<\varepsilon<|\theta-\alpha-a|$, we have

$$
\begin{aligned}
\left.V^{\prime}(t)\right|_{(1)}+\varepsilon V(t) \leq & -\left(\gamma-\Omega-\varepsilon+\eta x_{1}(t)\right) x_{1}(t)-(\theta-\alpha) x_{2}(t) \\
& +(a+\varepsilon) x_{2}(t)-\left(b+c x_{3}(t)-\varepsilon\right) x_{3}(t) .
\end{aligned}
$$

There exist $C>0$ such that $\left.V^{\prime}(t)\right|_{(1)}+\varepsilon V(t) \leq C$, then $\lim _{t \rightarrow+\infty} V(t) \leq$ $C / \varepsilon:=M_{1}^{*}$. Therefore, exist $T_{1}>0$ and constant $M_{1}>M_{1}^{*}$ such that $x_{i}(t)<$ $M_{1}(i=1,2,3)$ for all $t>T_{1}$. From the fourth equation of system (1), we have $y^{\prime}(t) \leq y(t)\left(k E M_{1}-d-f y(t)\right)$. Then, there exist $T_{2}>T_{1}$ and positive constant $M_{2}$ such that $y(t) \leq\left(k E M_{1}-d\right) / f:=M_{2}$ for all $t>T_{2}$.

Lemas 3. If condition $C_{2}$ holds, then (1) has no nontrivial periodic solutions of $\tau$.

Proof. Assume (1) has a nontrivial periodic solution of period $\tau$, then the following differential equations

$$
\left\{\begin{array}{l}
x_{1}^{\prime}(t)=\alpha x_{2}(t)-\left(\gamma+\eta x_{1}(t)+E y(t)\right) x_{1}(t) \\
x_{2}^{\prime}(t)=\Omega x_{1}(t)-\theta x_{2}(t) \\
x_{3}^{\prime}=a x_{2}(t)-b x_{3}(t)-c x_{3}^{2}(t) \\
y^{\prime}(t)=y(t)\left(k E x_{1}(t)-d-f y(t)\right)
\end{array}\right.
$$

have periodic solutions. Let $V(t)=\sum_{i=1}^{3} c_{i} x_{i}(t)+c_{4} y(t)$, where $c_{i}(i=1,2,3,4)$ are positive constants to be determined, it follows that

$$
\begin{aligned}
\left.V^{\prime}(t)\right|_{(10)}= & \sum_{i=1}^{3} c_{i} x_{i}^{\prime}(t)+c_{4} y^{\prime}(t) \\
= & \left(c_{2} \Omega-c_{1} \gamma\right) x_{1}(t)-c_{1} \eta x_{1}^{2}(t)+\left(c_{1} \alpha-c_{2} \theta+c_{3} a\right) x_{2}(t) \\
& -c_{3}\left(b+c x_{3}(t)\right) x_{3}(t)+E x_{1}(t) y(t)\left(c_{4} k-c_{1}\right) \\
& -c_{4} y(t)(d+f y(t)) .
\end{aligned}
$$

Let $c_{1}=1, c_{2}=\gamma / \Omega, c_{3}=(\alpha \Omega-\gamma \theta) /(a \Omega), c_{4}=1 / k$, then

$$
V^{\prime}(t)=-c_{1} \eta x_{1}^{2}(t)-c_{3}\left(b+c x_{3}(t)\right) x_{3}(t)-c_{4} y(t)(d+f y(t)) \leq 0 .
$$

Applying Barbalat's lemma [8], we conclude that

$$
\lim _{t \rightarrow \infty}\left(x_{1}(t), x_{2}(t), x_{3}(t), y(t)\right)=E_{2}=\left(x_{1}^{*}, x_{2}^{*}, x_{3}^{*}, y^{*}\right)
$$

which contradicts the fact that (1) has periodic solutions.

Theorem 4. Suppose $C_{2}, C_{3}$ and $q>0$ hold, then for each $\tau>\tau_{j}(j=$ $0,1,2, \cdots),(1)$ has at least $j+1$ periodic solutions, where $\tau_{j}$ is defined in (8). 

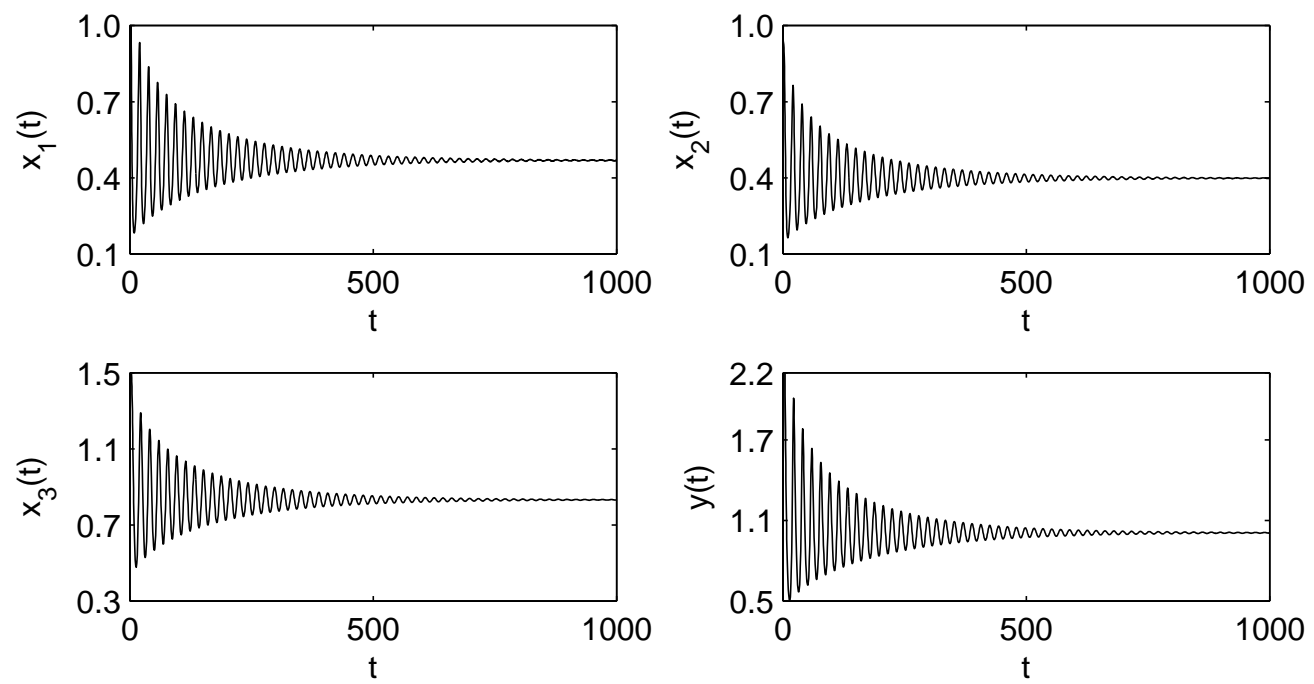

Figure 1: The time-series plot show that positive equilibrium point $E_{2}$ of system (1) is asymptotically stable for $\tau=2.3<\tau_{0}$.

Proof. The characteristic equation of (1) at the positive equilibrium $E_{2}$ is given by

$$
\operatorname{det}\left(\Delta\left(E_{2}, \tau, p\right)(\lambda)\right)=\left(\lambda+b+2 c x_{3}^{*}\right)\left(M(\lambda)+N(\lambda) e^{-\lambda \tau}\right) .
$$

The equilibrium $E_{2}$ is local stable when $\tau=0$, it can be verified that $\left(E_{2}, \tau_{j}\right.$, $\left.2 \pi / \omega_{0}\right)$ are isolated centers. Let

$$
\Omega_{\varepsilon, 2 \pi / \omega_{0}}=\left\{(\mu, p): 0<\mu<\varepsilon,\left|p-2 \pi / \omega_{0}\right|<\varepsilon\right\}
$$

Clearly, $\left|\tau-\tau_{j}\right| \leq \delta$ and $(\mu, p) \in \partial \Omega_{\varepsilon}$, then the necessary and sufficient conditions for $\Delta\left(E_{2}, \tau, p\right)(\mu+\mathrm{i} 2 \pi / p)=0$ are $\mu=0, \tau=\tau_{j}$ and $p=2 \pi / \omega_{0}$. Define

$$
H^{ \pm}\left(E_{2}, \tau_{j}, 2 \pi / \omega_{0}\right)(\mu, p)=\Delta\left(E_{2}, \tau_{j} \pm \delta, p\right)(\mu \pm \mathrm{i} 2 \pi / p)
$$

Then, we have the transversal number

$$
\begin{aligned}
\gamma\left(E_{2}, \tau_{j}, 2 \pi / \omega_{0}\right)= & \operatorname{deg}_{B}\left(H^{-}\left(E_{2}, \tau_{j}, 2 \pi / \omega_{0}\right), \Omega_{\varepsilon, 2 \pi / \omega_{0}}\right) \\
& -\operatorname{deg}_{B}\left(H^{+}\left(E_{2}, \tau_{j}, 2 \pi / \omega_{0}\right), \Omega_{\varepsilon, 2 \pi / \omega_{0}}\right)=-1
\end{aligned}
$$

By Theorem 3.3 of $\mathrm{Wu}$ [4], we conclude that the connected component $l_{\left(E_{2}, \tau_{j}, 2 \pi / \omega_{0}\right)}$ through $\left(E_{2}, \tau_{j}, 2 \pi / \omega_{0}\right)$ in $\Sigma$ is nonempty. Meanwhile, we have

$$
(z(t), \tau, p) \sum_{\left(E_{2}, \tau_{j}, 2 \pi / \omega_{0}\right)} \gamma(z(t), \tau, p)<0
$$



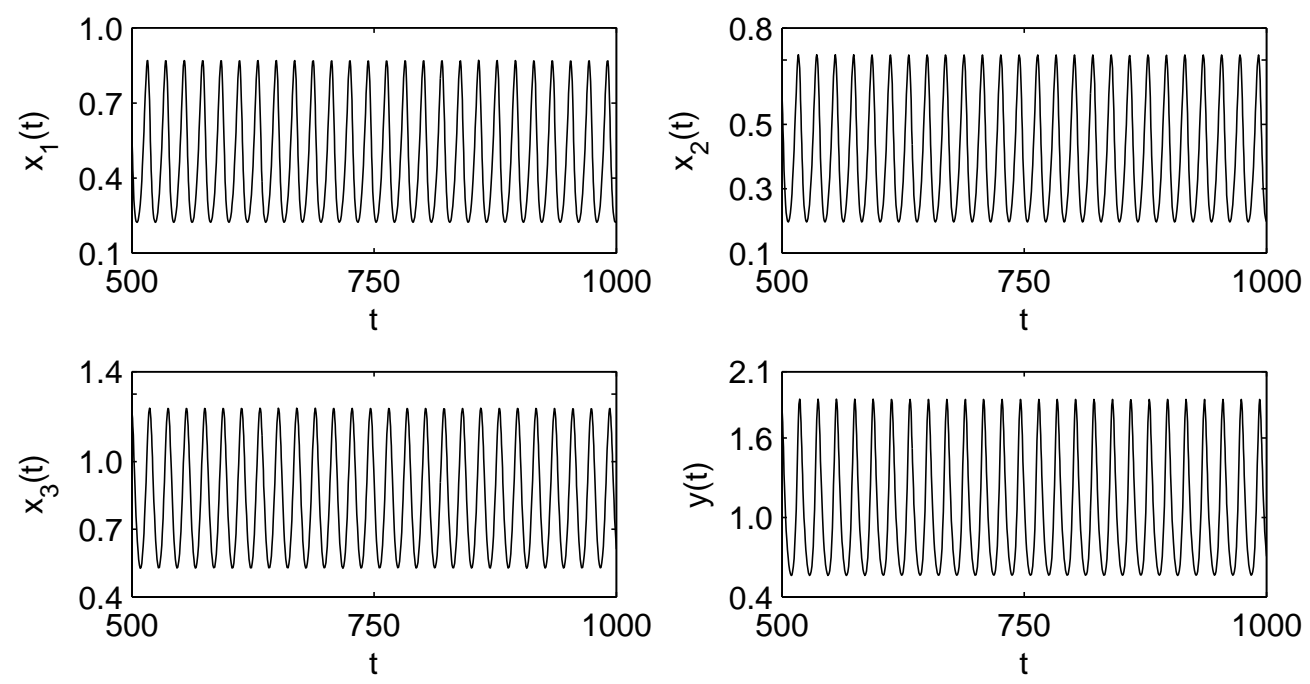

Figure 2: The time-series plot show that the local Hopf bifurcation for $\tau=2.5>\tau_{0}$ near positive equilibrium point $E_{2}$.

and hence $l_{\left(E_{2}, \tau_{j}, 2 \pi / \omega_{0}\right)}$ is unbounded.

Lemma 1 implies that the projection of $l_{\left(E_{2}, \tau_{j}, 2 \pi / \omega_{0}\right)}$ onto the $z$-space is bounded. From the definition of $\tau_{j}$, we have $2 \pi / \omega_{0}<\tau_{j}$ for $j \geq 1$. Lemma 2 implies that the projection of $l_{\left(E_{2}, \tau_{j}, 2 \pi / \omega_{0}\right)}$ onto the $\tau$-space is bounded. Assume the projection of $l_{\left(E_{2}, \tau_{j}, 2 \pi / \omega_{0}\right)}$ onto the $\tau$-space $\left(0, \tau^{*}\right)$ and $\tau^{*}>\tau_{j}$. Applying Lemma 2, we know $(z(t), \tau, p) \in l_{\left(E_{2}, \tau_{j}, 2 \pi / \omega_{0}\right)}$ implies $p<\tau_{j}$. This shows that in order for $l_{\left(E_{2}, \tau_{j}, 2 \pi / \omega_{0}\right)}$ to be unbounded, its projection onto the $\tau$-space must be unbounded. Consequently, the projection of $l_{\left(E_{2}, \tau_{j}, 2 \pi / \omega_{0}\right)}$ onto the $\tau$-space includes $\left[\tau_{j},+\infty\right)$. This shows that, for each $\tau_{j},(1)$ has $j+1$ nonconstant periodic solutions.

\section{Numerical Simulation}

Let $\alpha=2.8, \gamma_{1}=0.2, \Omega=0.85, \eta=0.15, E=1.25, \theta_{1}=0.2, a=0.8, b=$ $0.3, c=0.1, k=0.6, d=0.15, f=0.2$ and the initial point $X(0)=(1,1,1,1)$. System (1) has unique positive equilibrium point $E_{2}=(0.47,0.40,0.83,1.01)$. We evaluate $p=11.80, q=0.29, r=-0.20, \omega_{0}=0.3417, \tau_{0}=2.4084$ and $\tau_{j}=2.4084+j 5.854 \pi(j=0,1,2, \cdots)$. The the positive equilibrium point $E_{2}$ is asymptotically stable when $\tau=2.3<\tau_{0}$ (Fig. 1). When $\tau=2.5>\tau_{0}$, 

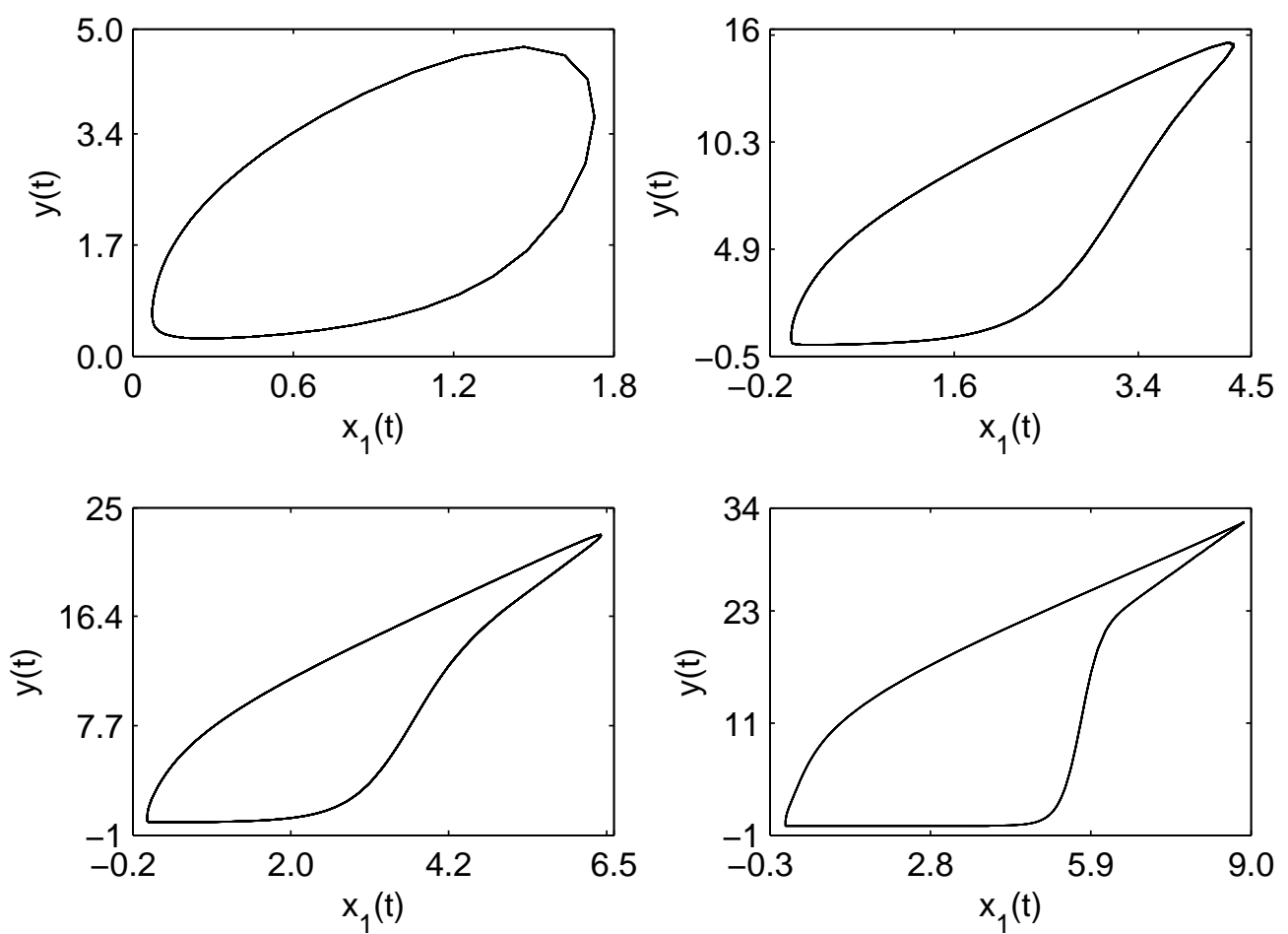

Figure 3: The large period oscillation (global Hopf bifurcation) of system (1) when $\tau=3,5,7,25$.

the positive equilibrium $E_{2}$ is unstable and the local Hopf bifurcation occurring around the positive $E_{2}$ are shown (Fig. 2). The simulations consistently show global existence of periodic solution (global Hopf bifurcation): existence of large amplitude periodic solutions for values of $\tau$ far away from $\tau_{j}$, where $\tau=$ $3,5,7,25$ and the periodic solutions are stable. From Fig. 3, we know that the period of the periodic solutions are increasing when $\tau 3$ to 25 . These are the slowly oscillating periodic solutions for (1). But, too large time delay would make the population to be die out, because the population very close to zero as time delay increase to some critical value.

\section{Conclusion}

In this paper, we studied a delayed stage-structured prey-predator system and analyzed the stability of the positive equilibrium, obtained the conditions of 
the positive equilibrium occurring local Hopf bifurcation and global Hopf bifurcation (global periodic solution).

Numerical examples by time-series plot, shown that the system considered local asymptotically stable and stable local Hopf bifurcation periodic solutions. And the populations can be coexistence with large periodic fluctuating under some conditions which caused by the large time delay far away from $\tau_{0}$, and the amplitudes of period oscillatory are increasing as time delays increased. In particular, we observe that the solutions of the delayed system could arbitrary close to zero when the delay tend to some critical values, which means that the prey or predator would tend to extinction. These are very interesting in mathematics and biology.

\section{Acknowledgements}

This work was supported by the The Natural Science Foundation of Guizhou Province (Nos. [2011]2116, LKQS[2013]01, LKQS[2013]12).

\section{References}

[1] S.Y. Li X.G. Xue. Hopf bifurcation in a three-stage-structured preypredator system with predator density dependent. Communications in Computer and Information Science, 288, (2012), 740-747.

[2] S.Y. Li, Y. Xue, W.W. Liu. Hopf bifurcation and global periodic solutions for a three-stage-structured prey-predator system with delays. International Journal of Information and Systems Sciences, 8, No. 1 (2012), $142-156$.

[3] C.J. Sun, M.A. Han, Y.P. Lin. Analysis of stability and Hopf bifurcation for a delayed logistic equation. Chaos, Solitons 85 Fractals, 31, No. 3 (2007), 672-682. doi:10.1016/j.chaos.2005.10.019.

[4] J.H. Wu. Symmetric functional differential equations and neural networks with memory. Transactions of the American Mathematical Society, 350, No. 12 (1998), 4799-4838.

[5] X.P. Yan, W.T. Li. Hopf bifurcation and global periodic solutions in a delayed predator-prey system. Appl. Math. Comp., 177, No. 1 (2006), 427445. doi:10.1016/j.amc.2005.11.020. 
[6] Z.H. Wang. A very simple criterion for characterizing the crossing direction of time-delayed systems with delay-dependent parameters. International Journal of Bifurcation and Chaos, 22, No. 3 (2012), 1250048 (7pages). doi: 10.1142/S0218127412500484.

[7] J.K. Hale. Theory of functional differential equations. Springer, New York (1977).

[8] K. Gopalsamy. Stability and oscillations in delay differential equations of population dynamics. Kluwer Academic, Dordrecht (1992). 